Copyright American Chemical Society

Supporting Information for Organic Letters

\title{
The First Bowl-Shaped Stable Neutral Radical with a Corannulene System: Synthesis and Characterization of the Electronic Structure
}

\author{
Yasushi Morita, Shinsuke Nishida, Tadahiro Kobayashi, Kozo Fukui, Kazunobu Sato, \\ Daisuke Shiomi, Takeji Takui, and Kazuhiro Nakasuji
}

\section{Supporting Information}

General. ${ }^{1} \mathrm{H}$ NMR and ${ }^{13} \mathrm{C}$ NMR spectra were recorded at $600 \mathrm{MHz}$ with $\mathrm{CDCl}_{3}$ as solvent and $\mathrm{Me}_{4} \mathrm{Si}$ or residual solvent as an internal standard. Infrared spectra were recorded using $\mathrm{KBr}$ plates. UV-vis spectra were recorded using $\mathrm{KBr}$ plates and $\mathrm{CH}_{3} \mathrm{CN}$ solution. The liquid-phase ESR, ENDOR, and TRIPLE spectra were recorded in toluene solutions at temperature range of $T$ $=270 \sim 293 \mathrm{~K}$ on Bruker ESR/ENDOR spectrometers ESP 300/350. The solution of the radical was degassed by freeze-pump-thaw method before the measurements. Cyclic voltammetric measurements were made with an ALS Electrochemical Analyzer Model 612A. Cyclic voltammograms were recorded with $1.6 \mathrm{~mm}$ diameter gold working electrode and $\mathrm{Pt}$ wire counter electrode in $\mathrm{CH}_{3} \mathrm{CN}$ containing $0.1 \mathrm{M} \mathrm{Bu}_{4} \mathrm{NClO}_{4}$ as a supporting electrolyte under argon atmosphere at room temperature. The experiments employed an $\mathrm{Ag} / 10 \mathrm{mM} \mathrm{AgNO}_{3}$ reference electrode and the final results were calibrated with the ferrocene/ferrocenium couple. EI-MS spectra were recorded at $70 \mathrm{eV}$. Melting points were measured with a hot-stage apparatus and are uncorrected. Elemental analyses were performed at the Graduate School of Science and Osaka University. $R_{f}$ values on TLC were recorded on E. Merck precoated $(0.25 \mathrm{~mm})$ silica gel $60 \mathrm{~F}_{254}$ plates. The plates were sprayed with a solution of $10 \%$ phosphomolybdic acid in $95 \% \mathrm{EtOH}$ and then heated until the spots became clearly visible. Alumina (200 mesh) was used for column chromatography. Solvents were dried (drying agent in parenthesis) and distilled under argon prior to use: THF (Na-benzophenone ketyl); DMF $\left(\mathrm{CaH}_{2}\right)$; toluene $\left(\mathrm{CaH}_{2}\right) ; \mathrm{CH}_{2} \mathrm{Cl}_{2}\left(\mathrm{CaH}_{2}\right)$; $\mathrm{CH}_{3} \mathrm{CN}\left(\mathrm{P}_{4} \mathrm{O}_{10}, \mathrm{CaH}_{2}\right)$. Active $\mathrm{PbO}_{2}{ }^{\mathrm{S} 1}$ was prepared and purified by the same procedure described in the literature. Corannulene, ${ }^{\mathrm{S} 2}$ bromocorannulene $^{\mathrm{S} 3}$ and 3-(4-methoxyphenyl)oxoverdazyl derivative $^{\mathrm{S} 4}$ were prepared by the procedures described in the literatures. All reactions requiring anhydrous conditions were conducted under argon atmosphere. 
2-Dibenzo[ghi,mno]fluoranthenecarbaldehyde (3) 2-Bromodibenzo[ghi,mno]fluoranthene (containing $5 \mathrm{~mol} \%$ of dibromo derivative, $100 \mathrm{mg}, 0.30 \mathrm{mmol}$ ) was placed in a $50-\mathrm{mL}$ Schlenk tube and dissolved with THF $(10 \mathrm{~mL})$ and stirred at $-78{ }^{\circ} \mathrm{C}$ under argon atmosphere for $1 \mathrm{~h}$. To the mixture was added $n$-BuLi ( $1.6 \mathrm{M}$ hexane solution, $0.38 \mathrm{~mL}, 0.61 \mathrm{mmol})$ and stirred for 15 min. To the mixture was added DMF $(0.13 \mathrm{~mL}, 1.8 \mathrm{mmol})$ and stirred for $6 \mathrm{~h}$. The reaction mixture was poured into a $\mathrm{pH} 7$ phosphate buffer solution $(0.1 \mathrm{M}, 20 \mathrm{~mL})$ and extracted with $\mathrm{CH}_{2} \mathrm{Cl}_{2}(50 \mathrm{~mL})$. The organic layer was washed with satd aqueous $\mathrm{NaCl}$ solution $(100 \mathrm{~mL})$ and water $(100 \mathrm{~mL})$, and dried over $\mathrm{Na}_{2} \mathrm{SO}_{4}$, then filtered and concentrated under reduced pressure, to give crude product as a bright yellow solid. The residual solid was subjected to deactivated silica gel column chromatography with a 20:1 1:1 mixture of hexane and $\mathrm{CH}_{2} \mathrm{Cl}_{2}$ as eluant, to give the formyl derivative $\mathbf{3}(31 \mathrm{mg}, 36 \%)$ as a light yellow powder. [If necessary, this product can be purified by sublimation in vacuo at $220{ }^{\circ} \mathrm{C}$.] mp $205-206^{\circ} \mathrm{C}$; TLC $\mathrm{R}_{f} 0.59$ (hexane/ethyl acetate = 2:1); ${ }^{1} \mathrm{H}$ NMR $\left(600 \mathrm{MHz}, \mathrm{CDCl}_{3}\right) \delta 7.82\left(\mathrm{~d}, J=8.8 \mathrm{~Hz}, \mathrm{H}^{6}, 1\right), 7.83\left(\mathrm{~d}, J=8.8 \mathrm{~Hz}, \mathrm{H}^{4}\right.$, 1), $7.84\left(\mathrm{~d}, J=8.8 \mathrm{~Hz}, \mathrm{H}^{3}, 1\right), 7.86\left(\mathrm{~d}, J=8.8 \mathrm{~Hz}, \mathrm{H}^{5}, 1\right), 7.87\left(\mathrm{~d}, J=8.8 \mathrm{~Hz}, \mathrm{H}^{7}, 1\right), 7.91(\mathrm{~d}, J=$ $\left.8.8 \mathrm{~Hz}, \mathrm{H}^{8}, 1\right), 7.91\left(\mathrm{~d}, J=8.8 \mathrm{~Hz}, \mathrm{H}^{2}, 1\right), 8.39\left(\mathrm{~s}, \mathrm{H}^{9}, 1\right), 8.69\left(\mathrm{~d}, J=8.8 \mathrm{~Hz}, \mathrm{H}^{1}, 1\right), 10.4\left(\mathrm{~s}, \mathrm{H}^{10}\right.$, 1); ${ }^{13} \mathrm{C}$ NMR $\left(600 \mathrm{MHz}, \mathrm{CDCl}_{3}\right) \delta 127.04\left(\mathrm{C}^{8}\right), 127.05\left(\mathrm{C}^{1}\right), 127.22\left(\mathrm{C}^{6}\right), 127.35\left(\mathrm{C}^{3}\right), 127.68$ $\left(C^{4}\right), 127.70,127.80\left(C^{7}\right), 128.69\left(C^{5}\right), 128.89\left(C^{2}\right), 128.92,130.95,131.28,132.50,135.05$, 135.20, 135.34, 136.143, 136.65, 138.26, $139.06\left(\mathrm{C}^{9}\right)$, $193.16\left(\mathrm{C}^{10}\right)$; IR $(\mathrm{KBr}) 2831,2710,1684$ $\mathrm{cm}^{-1}$; EI-MS, $m / z 278\left(\mathrm{M}^{+}, 31 \%\right), 249\left(\mathrm{M}^{+}-\mathrm{CHO}, 43 \%\right)$; Anal. Calcd for $\mathrm{C}_{21} \mathrm{H}_{10} \mathrm{O}: \mathrm{C} 90.63, \mathrm{H}$ 3.62, N 0.00. Found: C 90.24, H 3.54, N 0.00 . 
3-(Dibenzo[ghi,mno]fluoranthen-2-yl)-1,5-dimethyl-perhydro-1,2,4,5-tetrazin-6-one (4) 2Dibenzo[ghi,mno]fluoranthenecarbaldehyde (3) $(85.0 \mathrm{mg}, 0.305 \mathrm{mmol})$ was placed in a $20-\mathrm{mL}$ round-bottomed flask and dissolved with $\mathrm{CH}_{2} \mathrm{Cl}_{2}(4.6 \mathrm{~mL})$ under argon atmosphere. To the mixture were added 2,4-dimethylcarbonohydrazide (144 mg, $1.22 \mathrm{mmol}$ ) and a catalytic amount of $p-\mathrm{TsOH} \cdot \mathrm{H}_{2} \mathrm{O}$. The mixture was stirred at room temperature for $3 \mathrm{~h}$. The reaction mixture was poured into a pH 7 phosphate buffer solution $(0.1 \mathrm{M}, 20 \mathrm{~mL})$. To the mixture was added $\mathrm{CH}_{2} \mathrm{Cl}_{2}$ $(30 \mathrm{~mL})$. The mixture was washed with water $(30 \mathrm{~mL}$ x 3$)$. The organic layer was separated and dried over $\mathrm{Na}_{2} \mathrm{SO}_{4}$, then filtered and concentrated under reduced pressure and dried in vacuo. The residual solid was subjected to alumina column chromatography with a 3:1 mixture of ethyl acetate and ethanol as eluent, to give $4(90.6 \mathrm{mg}, 79 \%)$ as a pale yellow powder. $\mathrm{mp} 197-198{ }^{\circ} \mathrm{C}$; TLC $\mathrm{R}_{f} 0.57$ (THF); ${ }^{1} \mathrm{H}$ NMR $\left(600 \mathrm{MHz}, \mathrm{CDCl}_{3}\right) \delta 3.29\left(\mathrm{~s}, \mathrm{H}^{13,14}, 6\right), 4.73\left(\mathrm{~d}, J=10.7 \mathrm{~Hz}, \mathrm{H}^{11,12}\right.$, 2), $5.58\left(\mathrm{dt}, J=10.5\right.$ and $\left.1.4 \mathrm{~Hz}, \mathrm{H}^{10}, 1\right), 7.78\left(\mathrm{~d}, J=8.8 \mathrm{~Hz}, \mathrm{H}^{8}, 1\right), 7.80\left(\mathrm{~d}, J=8.8 \mathrm{~Hz}, \mathrm{H}^{6}, 1\right)$, $7.81\left(\mathrm{~d}, J=8.8 \mathrm{~Hz}, \mathrm{H}^{3}, 1\right), 7.82\left(\mathrm{~d}, J=8.2 \mathrm{~Hz}, \mathrm{H}^{5}, 1\right), 7.82\left(\mathrm{~d}, J=8.2 \mathrm{~Hz}, \mathrm{H}^{7}, 1\right), 7.83(\mathrm{~d}, J=8.5$ $\left.\mathrm{Hz}, \mathrm{H}^{6}, 1\right), 7.85\left(\mathrm{~d}, J=8.8 \mathrm{~Hz}, \mathrm{H}^{2}, 1\right), 7.90\left(\mathrm{~d}, J=1.4 \mathrm{~Hz}, \mathrm{H}^{9}, 1\right), 8.20\left(\mathrm{~d}, J=8.8 \mathrm{~Hz}, \mathrm{H}^{1}, 1\right) ;{ }^{13} \mathrm{C}$ NMR $\left(600 \mathrm{MHz}, \mathrm{CDCl}_{3}\right) \delta 38.15\left(\mathrm{C}^{13,14}\right), 68.41,124.19\left(\mathrm{C}^{9}\right), 126.10\left(\mathrm{C}^{1}\right), 126.95\left(\mathrm{C}^{8}\right), 126.98$ $\left(C^{3}\right), 127.14\left(C^{6}\right), 127.33\left(C^{2}\right), 127.44,127.46,127.49,129.06,130.09,130.71,130.91,131.23$, 133.21, 135.54, 135.91, 135.97, 136.06, $156.04\left(\mathrm{C}^{15}\right)$; $\mathrm{A}{ }^{13} \mathrm{C}$ signal was not observed due to overlapping with another signal; IR (KBr) 3227, 3024, 2925, $1623 \mathrm{~cm}^{-1}$; UV $\left(\mathrm{CH}_{3} \mathrm{CN}\right) \lambda_{\max }, \mathrm{nm}$ $(\varepsilon): 222\left(4.5 \times 10^{4}\right), 253\left(7.0 \times 10^{4}\right), 288\left(3.6 \times 10^{4}\right)$; EI-MS, $m / z 378\left(\mathrm{M}^{+}, 15 \%\right), 249\left(\mathrm{M}^{+}-\right.$ $\left.\mathrm{C}_{4} \mathrm{H}_{9} \mathrm{ON}_{4}, 100 \%\right)$; HRMS-EI $(m / z)$ : $\mathrm{M}^{+}$calcd for $\mathrm{C}_{24} \mathrm{H}_{18} \mathrm{~N}_{4} \mathrm{O}$, 378.1482; found, 378.1475; Anal. Calcd for $\mathrm{C}_{24} \mathrm{H}_{18} \mathrm{~N}_{4} \mathrm{O}$ : C 76.17, H 4.79, N 14.81. Found: C 75.72, H 4.82, N 14.49. 
6-(Dibenzo[ghi,mno]fluoranthen-2-yl)-2,3,4-trihydro-2,4-dimethyl-3-oxo-1,2,4,5-tetrazinyl (2) A radical precursor $4(10.0 \mathrm{mg}, 0.026 \mathrm{mmol})$ was placed in a $30-\mathrm{mL}$ round-bottomed flask and dissolved with $\mathrm{CH}_{2} \mathrm{Cl}_{2}(2.0 \mathrm{~mL})$ under argon atmosphere. To the mixture was added active $\mathrm{PbO}_{2}(25.3 \mathrm{mg}, 0.11 \mathrm{mmol})$ and stirred at room temperature for $1 \mathrm{~h}$. The resulting solid was filtered through the celite column, rinsed with $\mathrm{CH}_{2} \mathrm{Cl}_{2}$. The filtrates were concentrated under reduced pressure, to give 6-oxoverdazyl derivative $2(9.7 \mathrm{mg}, 98 \%)$ as a vivid red solid. $\mathrm{mp}$ $159-160{ }^{\circ} \mathrm{C}$ (dec); TLC $R_{f} 0.73$ (THF); IR (KBr) 3031, 2931, $1685 \mathrm{~cm}^{-1} ; \mathrm{UV}\left(\mathrm{CH}_{3} \mathrm{CN}\right) \lambda_{\max }, \mathrm{nm}$ (ع): $224\left(3.3 \times 10^{4}\right), 255\left(3.4 \times 10^{4}\right), 292\left(2.3 \times 10^{4}\right)$; EI-MS, $m / z 375\left(\mathrm{M}^{+}, 31 \%\right), 249\left(\mathrm{M}^{+}-\right.$ $\left.\mathrm{C}_{4} \mathrm{H}_{6} \mathrm{~N}_{4} \mathrm{O}, 16 \%\right), 275\left(\mathrm{M}^{+}-\mathrm{C}_{3} \mathrm{H}_{6} \mathrm{~N}_{3} \mathrm{O}, 100 \%\right)$; HRMS-EI $(\mathrm{m} / \mathrm{z}): \mathrm{M}^{+}$calcd for $\mathrm{C}_{24} \mathrm{H}_{15} \mathrm{~N}_{4} \mathrm{O}$, 375.1248; found, 375.1235. Anal. Calcd for $\left(\mathrm{C}_{24} \mathrm{H}_{15} \mathrm{~N}_{4} \mathrm{O}\right)\left(\mathrm{CH}_{2} \mathrm{Cl}_{2}\right)_{0.45}: \mathrm{C}$ 71.00, H 3.87, N 13.55. Found: C 71.00, H 3.90, N 13.56 . 
Table S1. UV-vis absorptions for $\mathbf{1 , 2 , 4}$ and $\mathbf{5}^{a}$

\begin{tabular}{cccccl}
\hline compd & $\lambda_{\max }$ & $\varepsilon / 10^{4}$ & compd & $\lambda_{\max }$ & $\varepsilon / 10^{4}$ \\
\hline $\mathbf{1}$ & 221 & 4.0 & $\mathbf{4}$ & 222 & 4.5 \\
& 251 & 6.0 & & 253 & 7.0 \\
& 286 & 3.1 & & 288 & 3.6 \\
$\mathbf{2}$ & 224 & 3.3 & $\mathbf{5}$ & 205 & 1.7 \\
& 255 & 3.4 & & 260 & 1.9 \\
& 292 & 2.3 & & 417 & 0.1 \\
\hline
\end{tabular}

${ }^{a} \mathbf{1}\left(1.1 \times 10^{-5} \mathrm{M}\right), \mathbf{2}\left(1.4 \times 10^{-5} \mathrm{M}\right), \mathbf{4}\left(1.0 \times 10^{-5} \mathrm{M}\right)$ and $\mathbf{5}\left(1.1 \times 10^{-5} \mathrm{M}\right)$ in $\mathrm{CH}_{3} \mathrm{CN}$ solutions. 


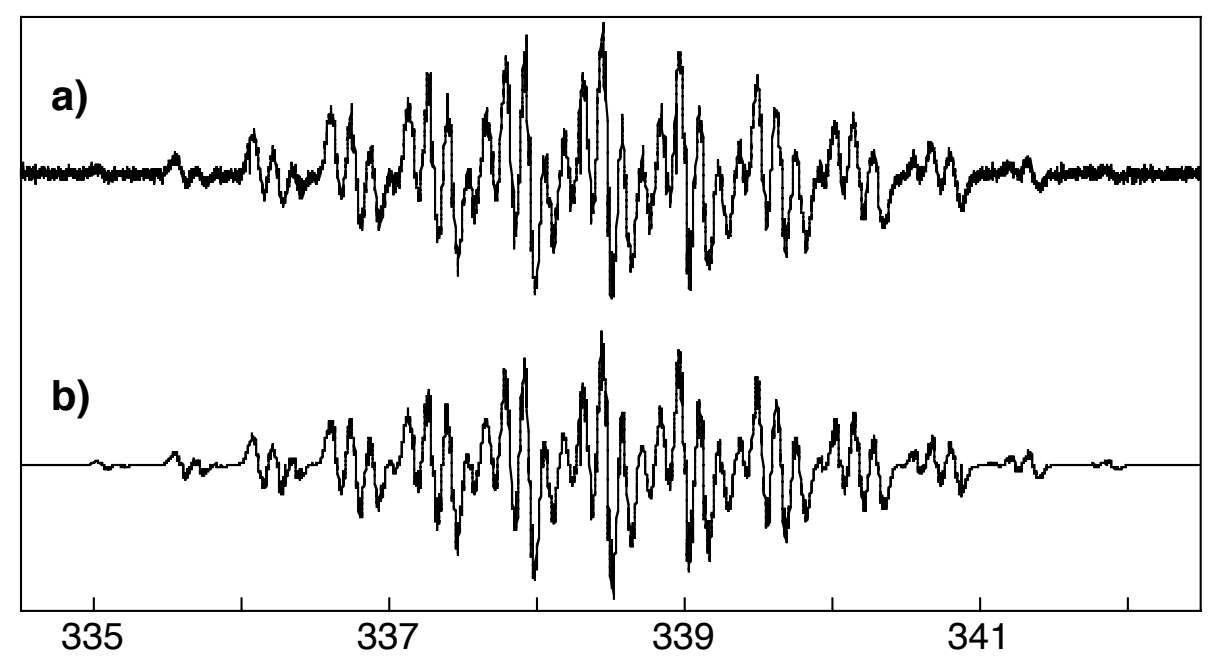

Field/mT

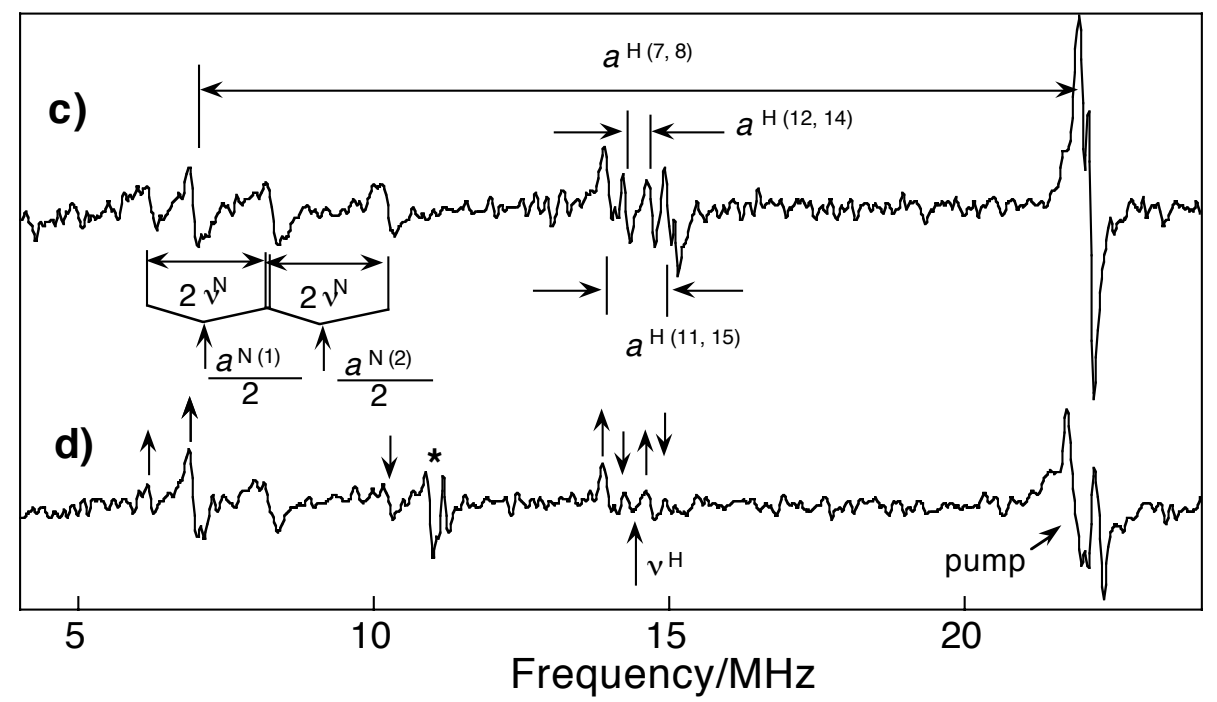

Figure S1. (a) Observed hyperfine ESR spectrum at $290 \mathrm{~K}$ (microwave frequency $9.498382 \mathrm{GHz}$ ), (b) a simulated spectrum, (c) ENDOR spectrum at $290 \mathrm{~K}$ and (d) TRIPLE spectrum at $290 \mathrm{~K}$ (pump frequency, $21.98 \mathrm{MHz}$. The asterisk shows artifacts of the spectrometer) for 5 in a toluene solution $\left(1.0 \times 10^{-4} \mathrm{M}\right)$. Observed $g$ value is 2.0041 . 


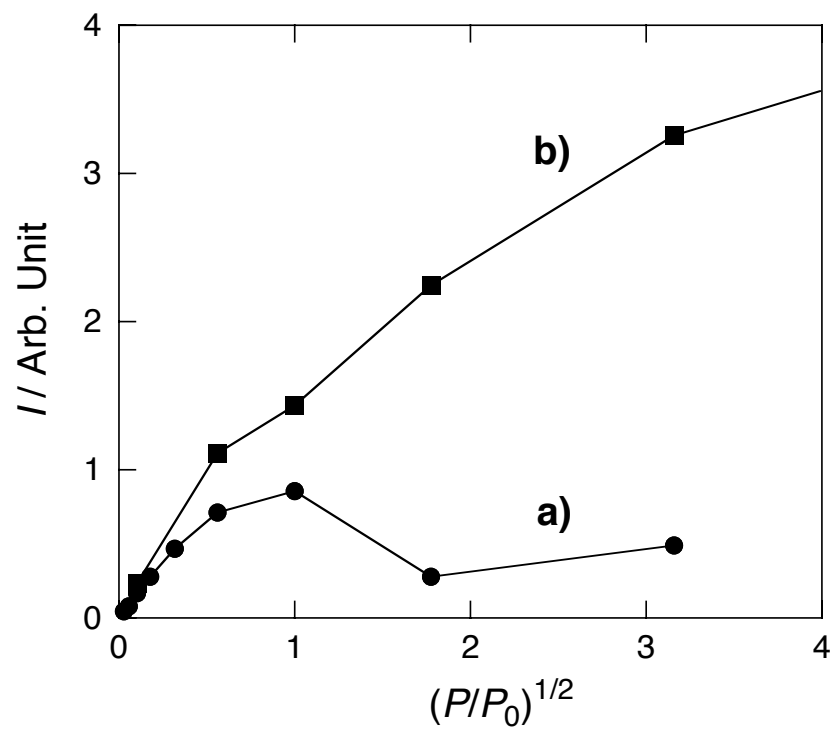

Figure S2. Microwave power dependence of the observed ESR intensities for $\mathbf{2}$ (a) and $\mathbf{5}$ (b), showing that the ESR absorption of $\mathbf{2}$ is readily saturated at the lower microwave power as compared to that of $\mathbf{5}$. 


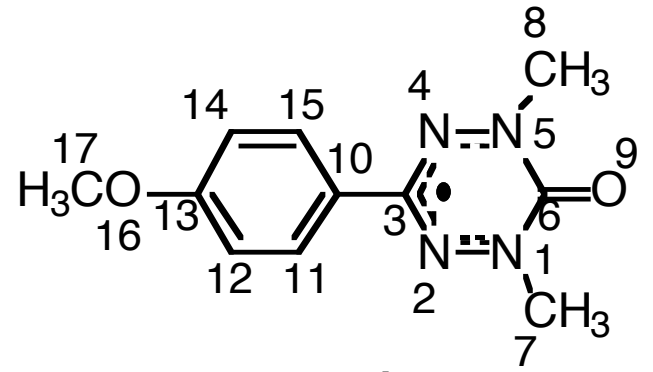

a)

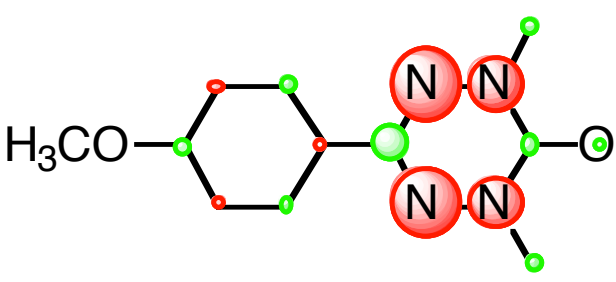

b)

Figure S3. (a) Atomic number of 5, (b) spin density distribution of $\mathbf{5}$ calculated by the DFT method. Green-colored and red-colored circles denote negative and positive spin densities. 
Table S2. Observed and calculated proton and nitrogen hfecs for $\mathbf{5}^{a}$

\begin{tabular}{|c|c|c|c|c|}
\hline \multicolumn{5}{|c|}{$a / \mathrm{mT}$} \\
\hline $\mathrm{N}(1,5)$ & $\mathrm{N}(2,4)$ & $\mathrm{H}(7,8)$ & $\mathrm{H}(11,15)$ & $\mathrm{H}(12,14)$ \\
\hline$+0.515^{b}$ & $+0.654^{b}$ & $+0.540^{b}$ & $+0.036^{b}$ & $-0.015^{b}$ \\
\hline $\pm 0.384^{c}$ & $\pm 0.546^{c}$ & $\pm 0.542^{c}$ & $+0.037^{c}$ & $-0.018^{c}$ \\
\hline
\end{tabular}



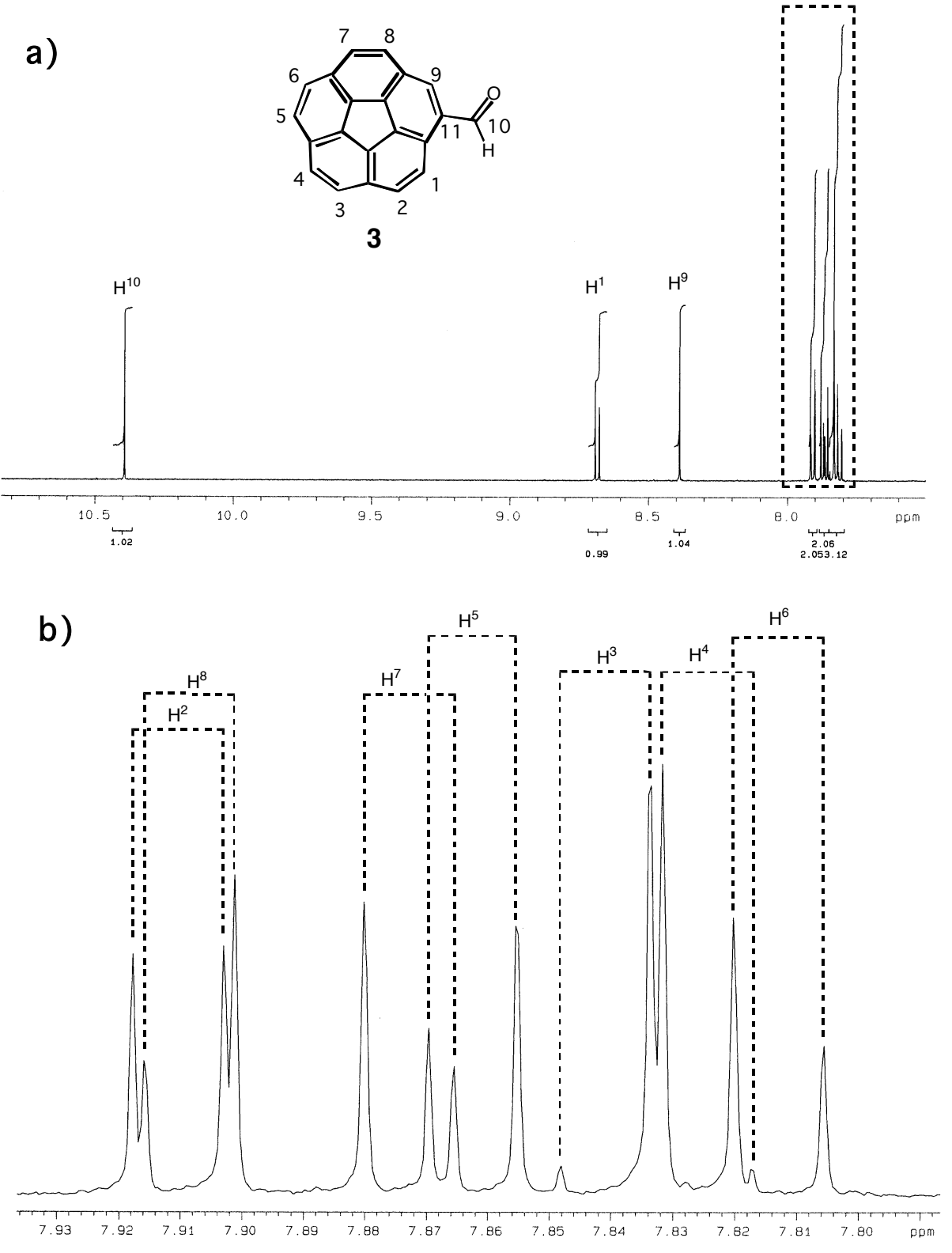

Figure S4. ${ }^{1} \mathrm{H}$ NMR $(600 \mathrm{MHz})$ spectra for $3\left(\mathrm{CDCl}_{3}\right.$ solution) at room temperature. (a) aromatic region; (b) magnified region between 7.80 and $7.99 \mathrm{ppm}$. 


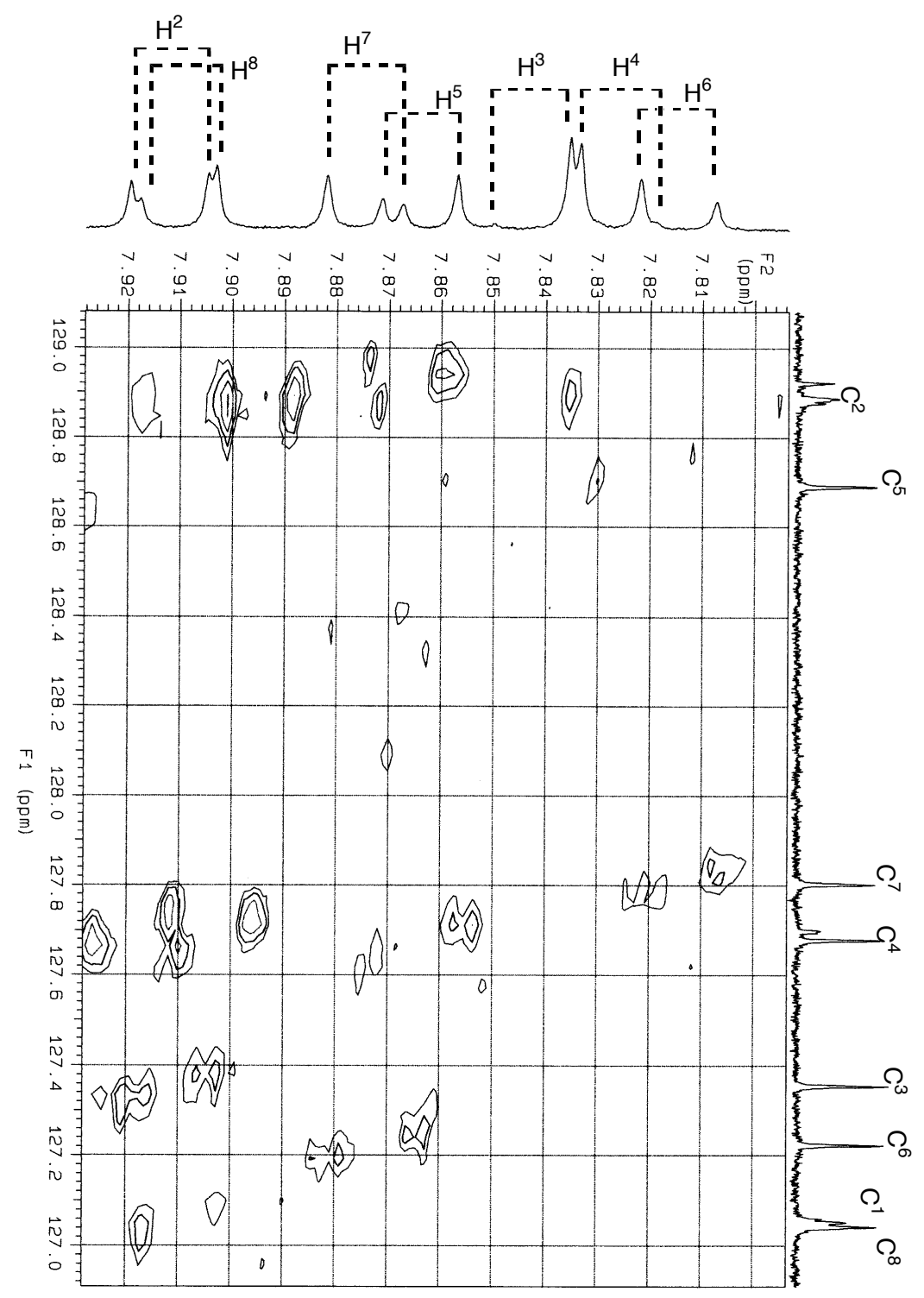

Figure S5. HMQC (600 MHz) spectrum for $3\left(\mathrm{CDCl}_{3}\right.$ solution) at room temperature. 


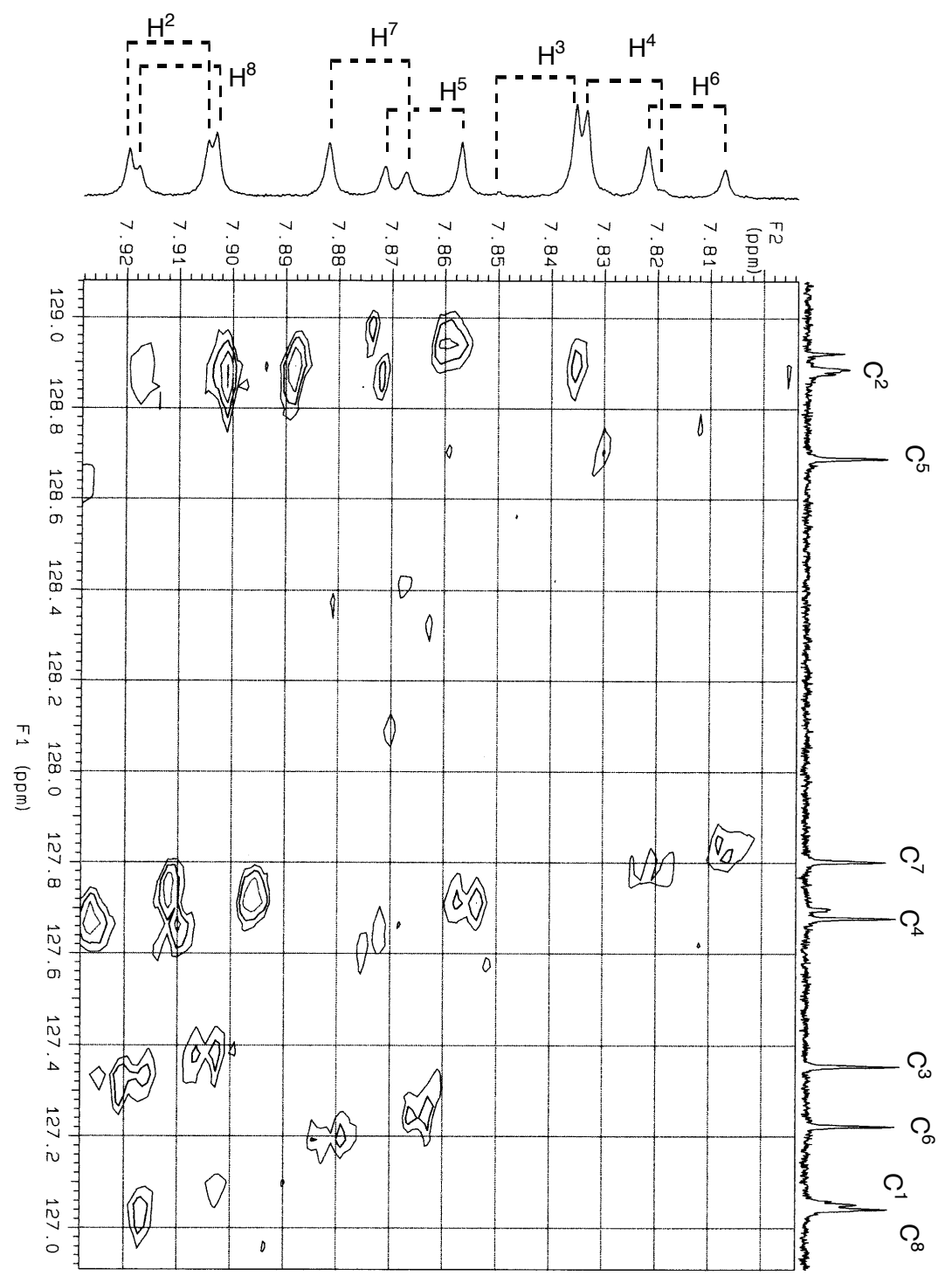

Figure S6. HMBC (600 MHz) spectrum for $\mathbf{3}\left(\mathrm{CDCl}_{3}\right.$ solution) at room temperature. 


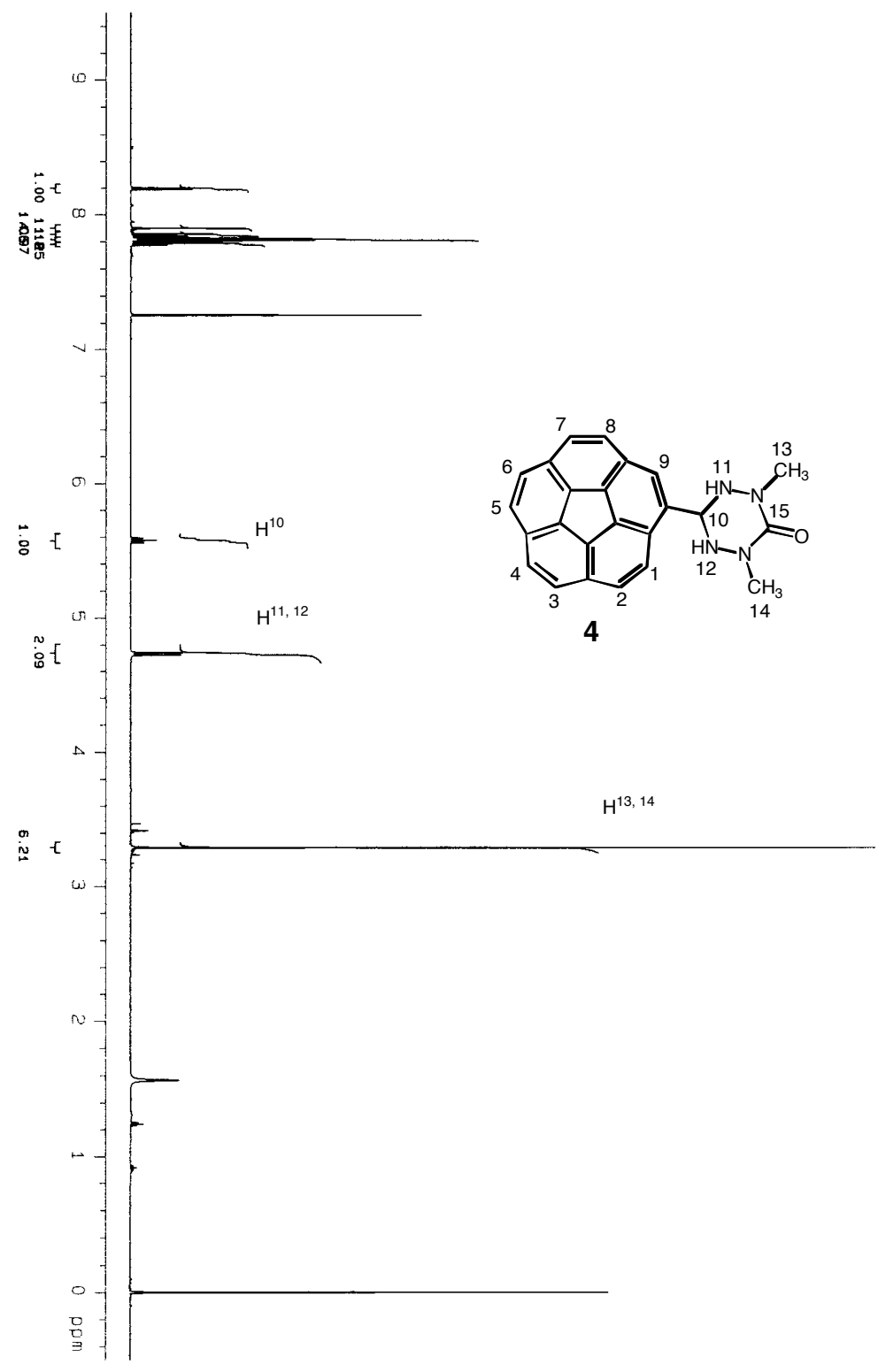

Figure S7. ${ }^{1} \mathrm{H}$ NMR $(600 \mathrm{MHz})$ spectra for $4\left(\mathrm{CDCl}_{3}\right.$ solution $)$ at room temperature. 

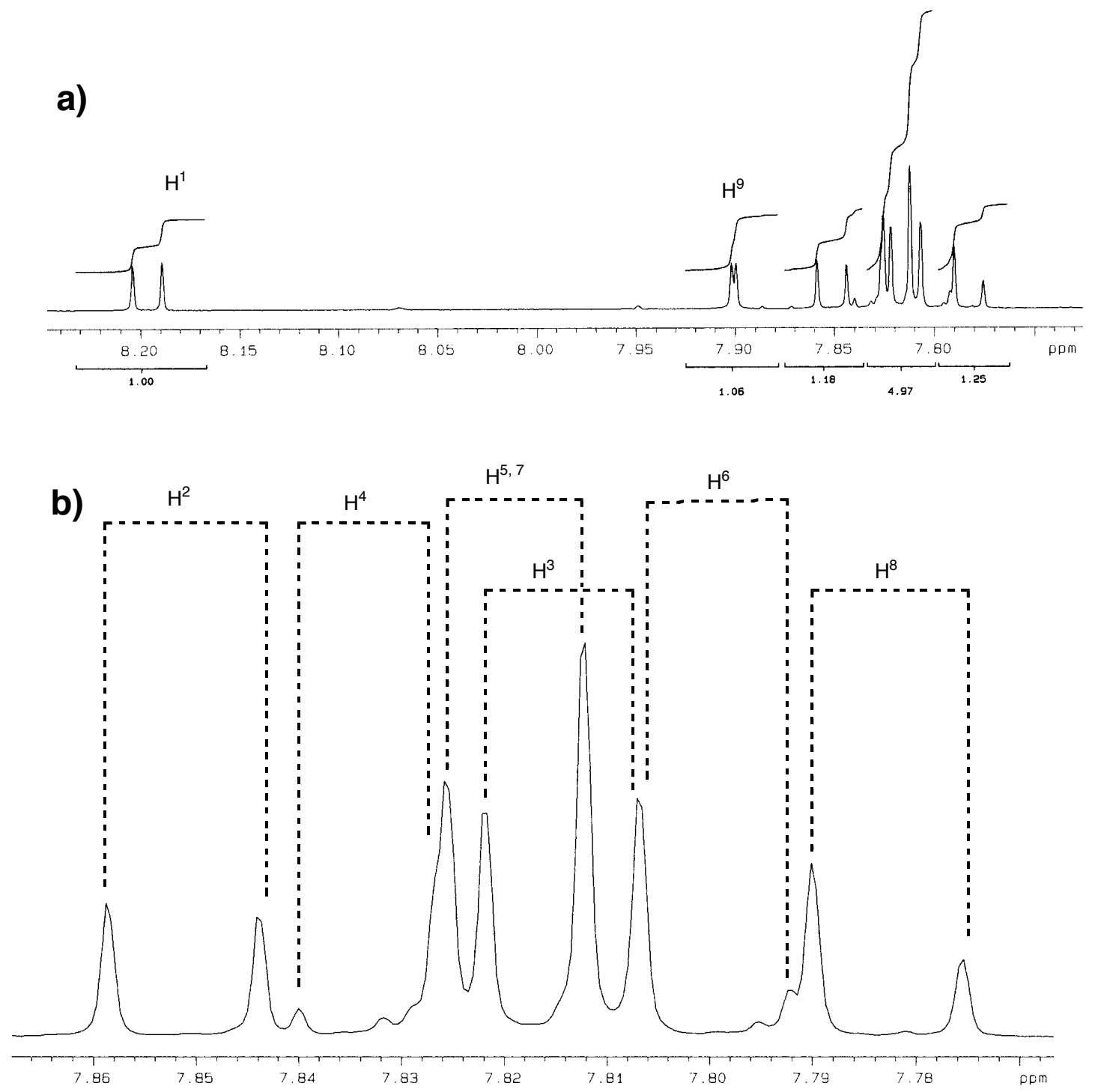

Figure S8. ${ }^{1} \mathrm{H} \mathrm{NMR}(600 \mathrm{MHz})$ spectra for $4\left(\mathrm{CDCl}_{3}\right.$ solution) at room temperature. a) aromatic region; b) magnified region between 7.77 and $7.87 \mathrm{ppm}$. 


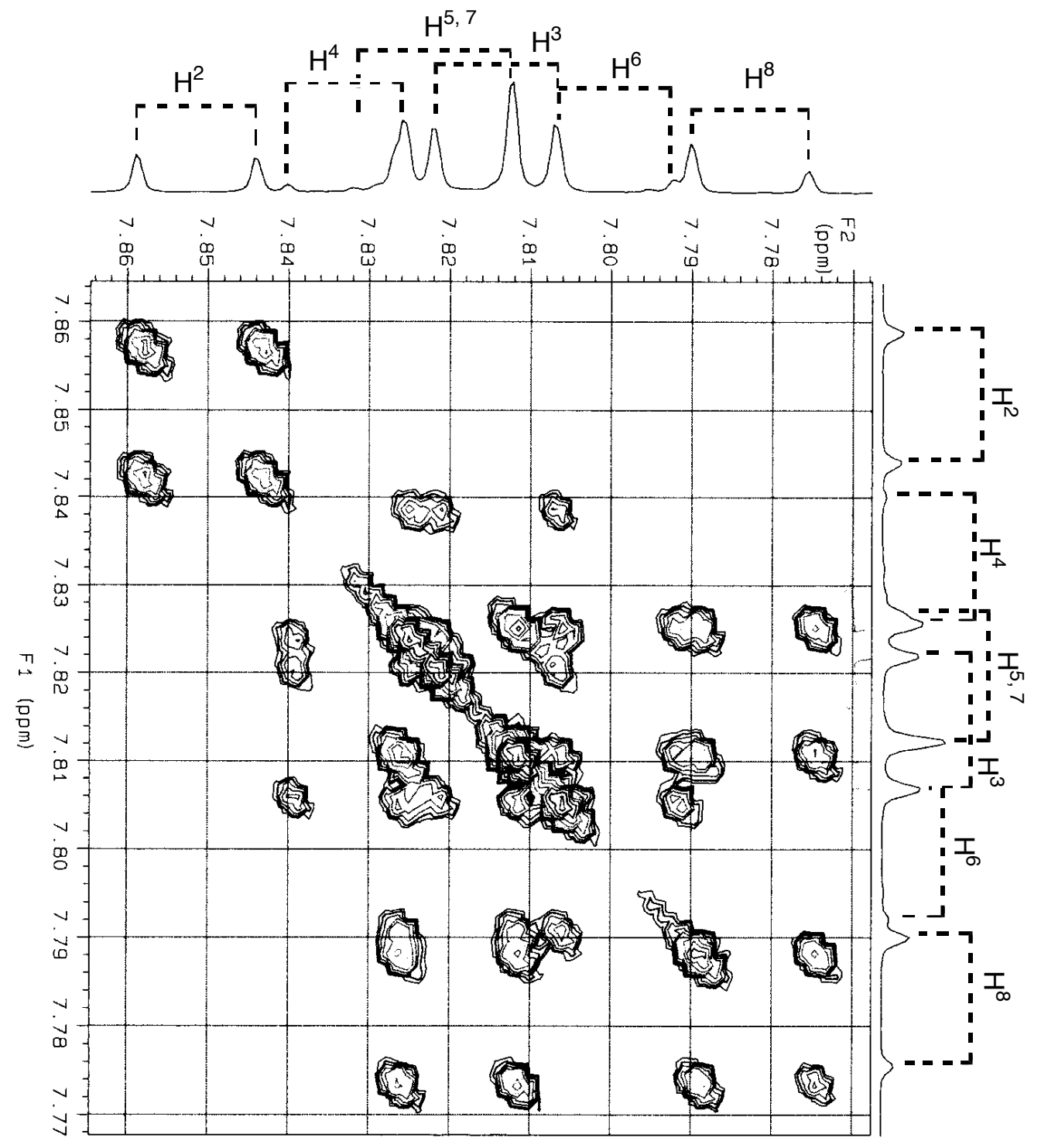

Figure S9. COSY $(600 \mathrm{MHz})$ spectrum for $4\left(\mathrm{CDCl}_{3}\right.$ solution $)$ at room temperature. 


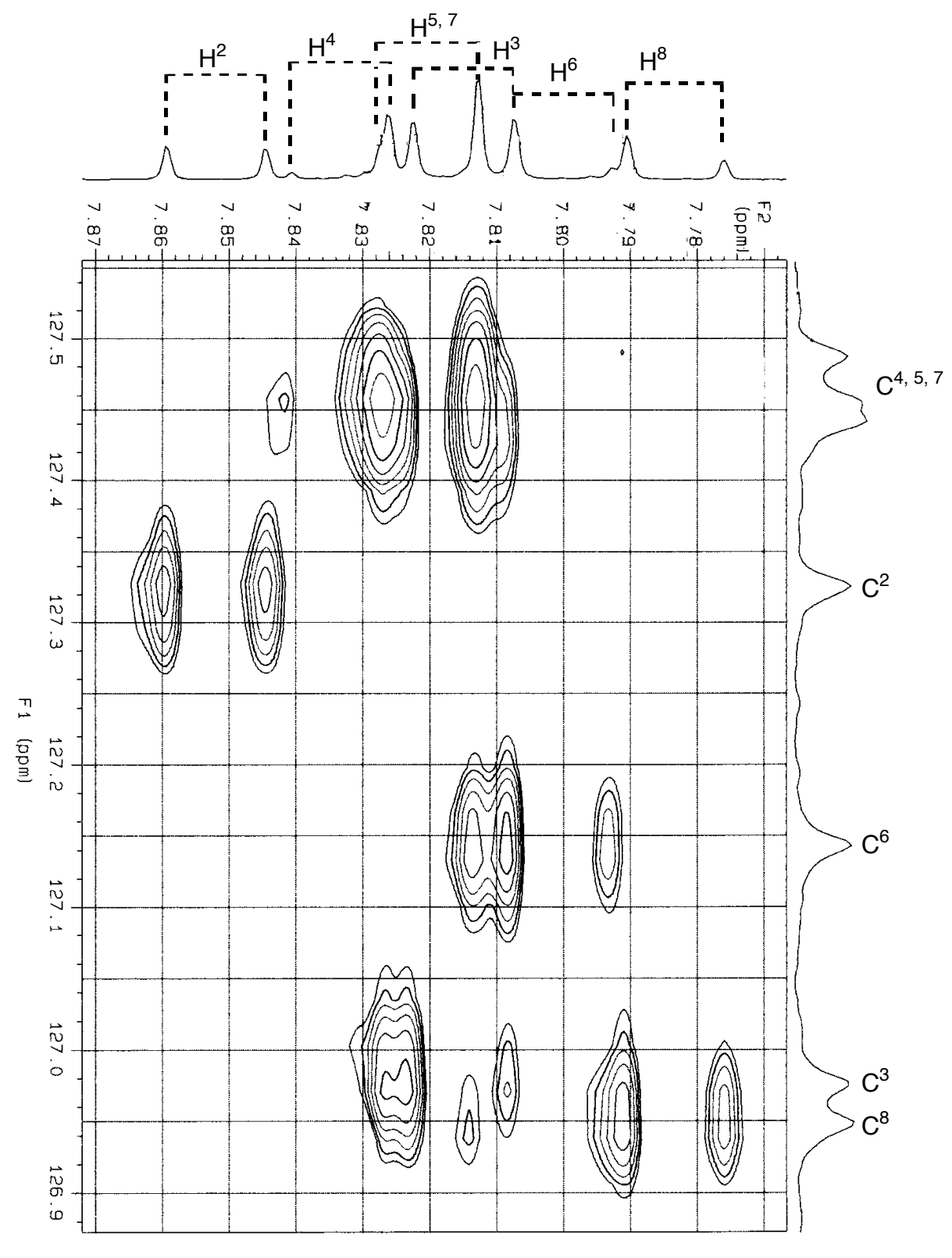

Figure S10. HMQC (600 MHz) spectrum for $4\left(\mathrm{CDCl}_{3}\right.$ solution) at room temperature. 


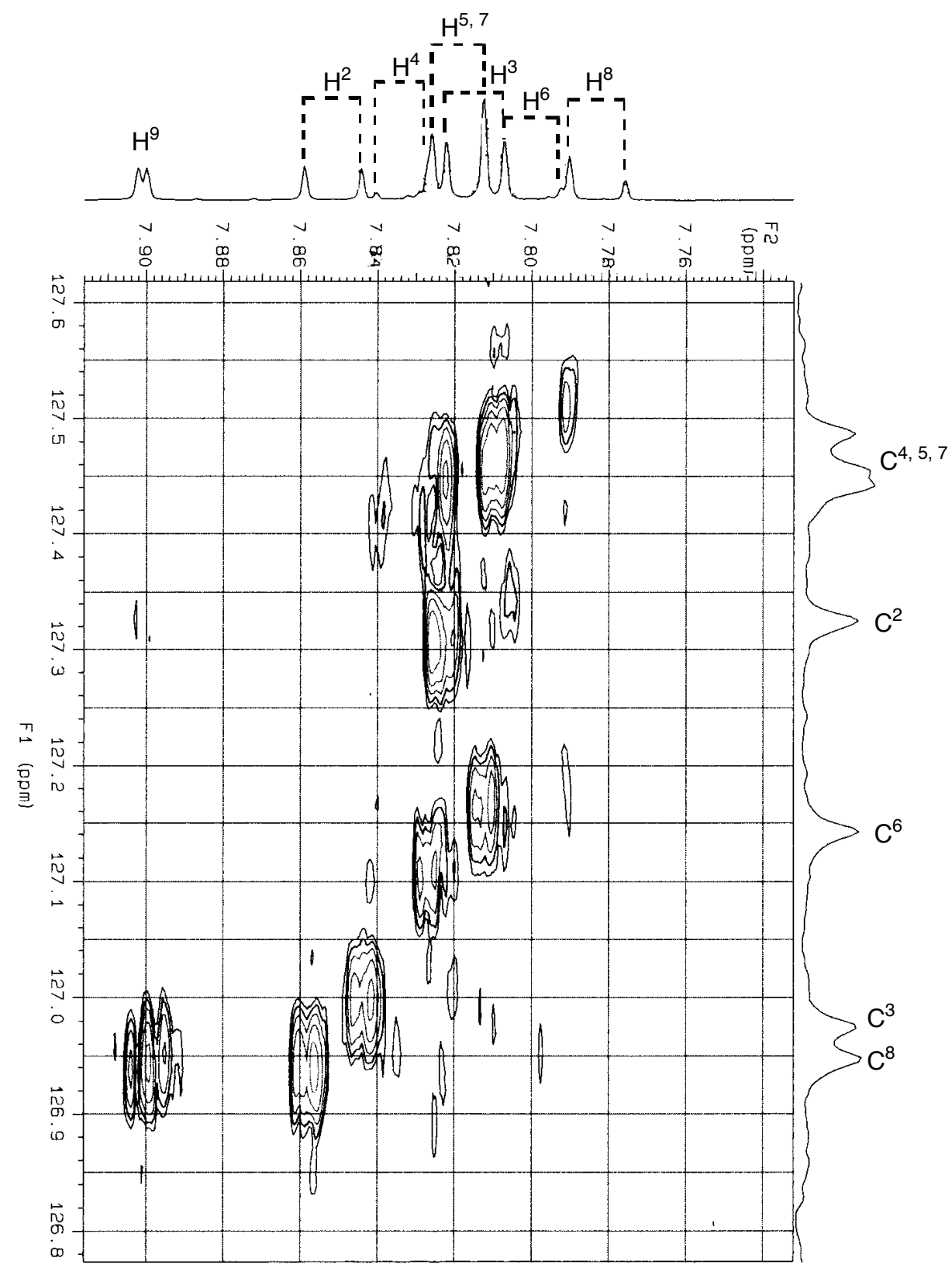

Figure S11. HMBC (600 MHz) spectrum for $4\left(\mathrm{CDCl}_{3}\right.$ solution) at room temperature. 


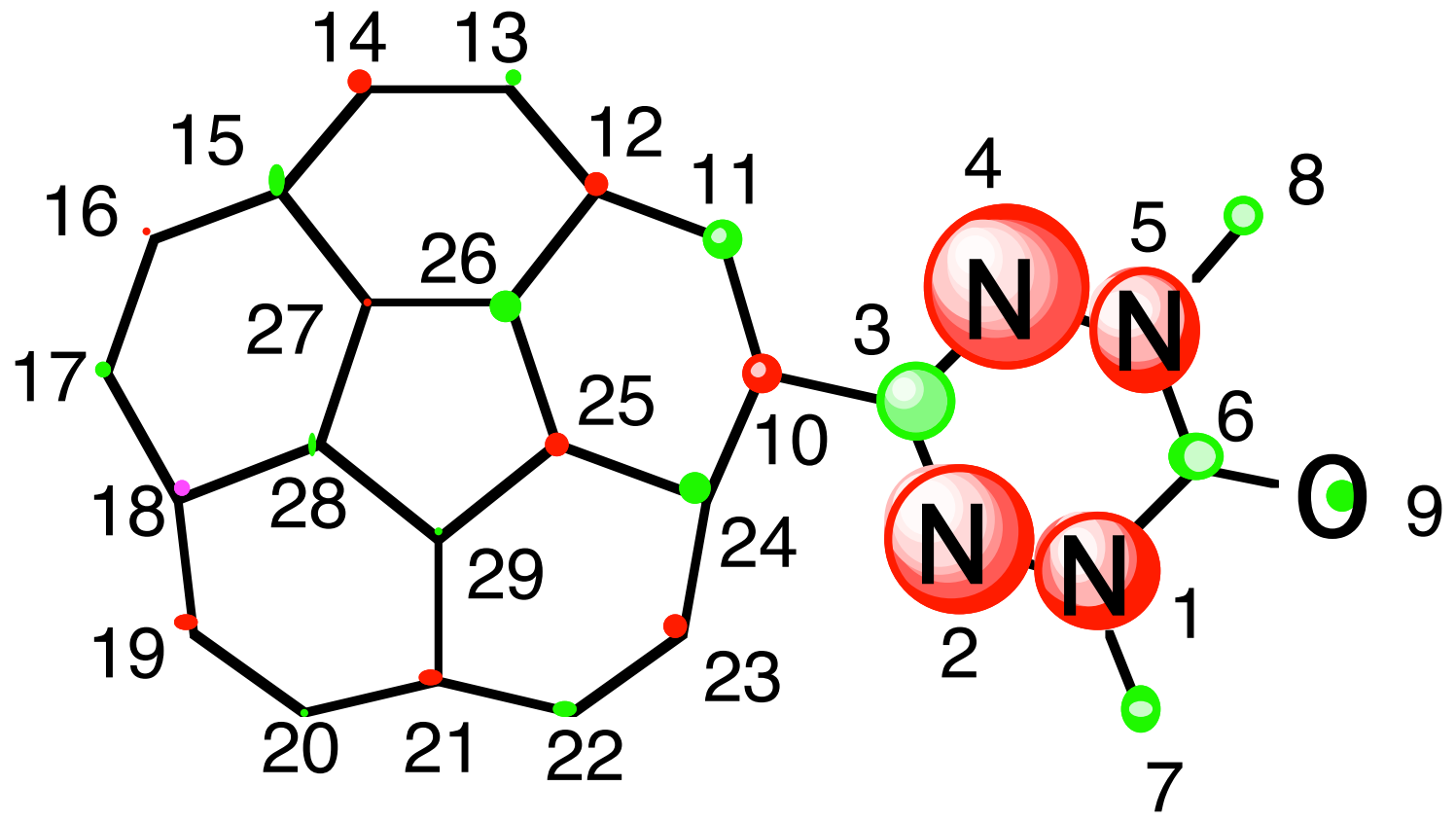

Figure S12. Spin density distribution of 2 calculated by using Gaussian 98 with UBLYP/6-31G**//UBLYP/6-31G** method. Green-colored and red-colored circles denote negative and positive spin density. N1, N5, +0.2047; N2, N4, +0.3466; C3, -0.0860 ; C6, -0.0244; C7, C8, -0.0157; O9, -0.0116; C10, +0.0162; C11, -0.0210 ; C12, $+0.0053 ; \mathrm{C} 13,-0.0030 ; \mathrm{C} 14,+0.0023 ; \mathrm{C} 15,-0.0032 ; \mathrm{C} 16,+0.0011 ; \mathrm{C} 17,-0.0025 ; \mathrm{C} 18$, $+0.0004 ; \mathrm{C} 19,+0.0010 ; \mathrm{C} 20,-0.0003 ; \mathrm{C} 21,+0.0018 ; \mathrm{C} 22,-0.0020 ; \mathrm{C} 23,+0.0040 ; \mathrm{C} 24$, -0.0063 ; C25, +0.0047; C26, $-0.0081 ; \mathrm{C} 27,+0.0020 ; \mathrm{C} 28,-0.0008 ; \mathrm{C} 29,-0.0011$. 


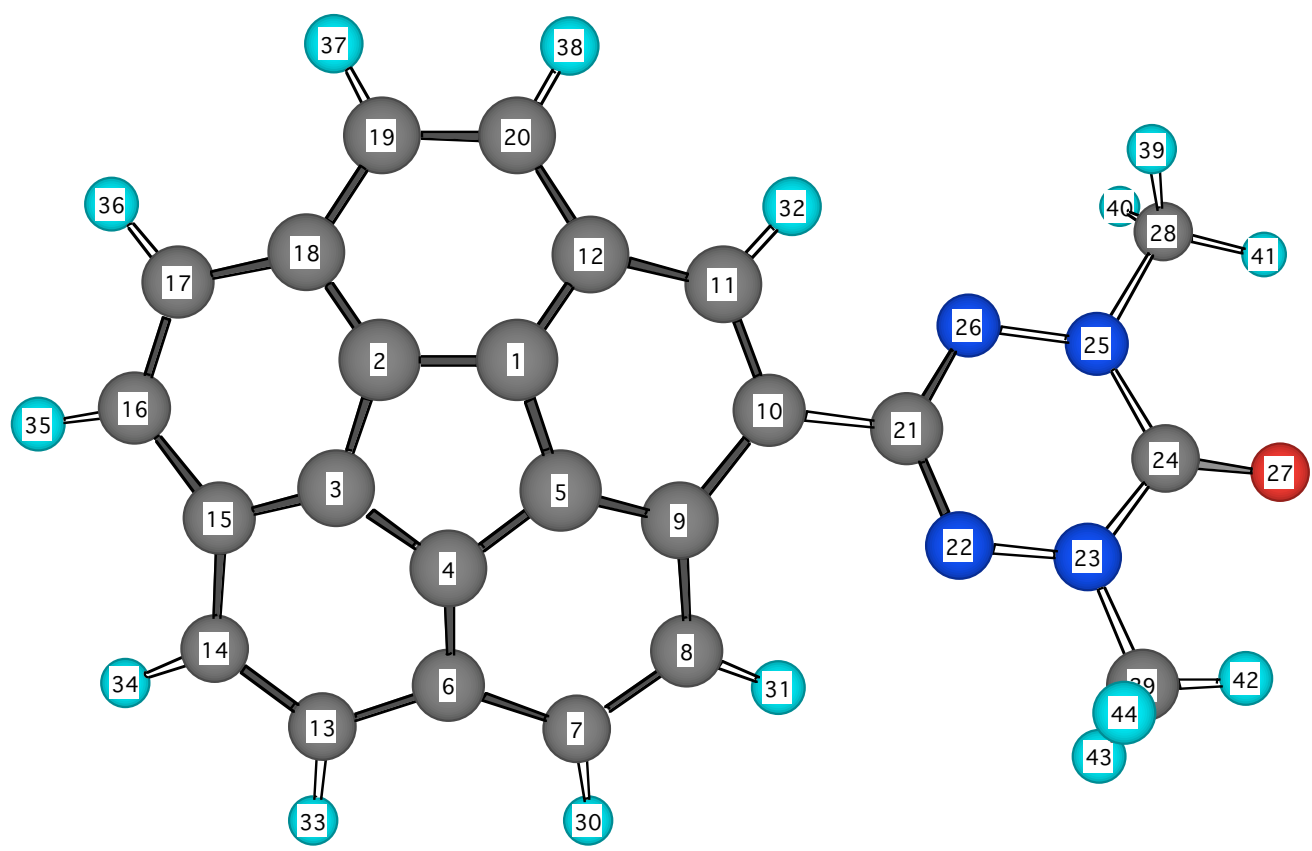

a)

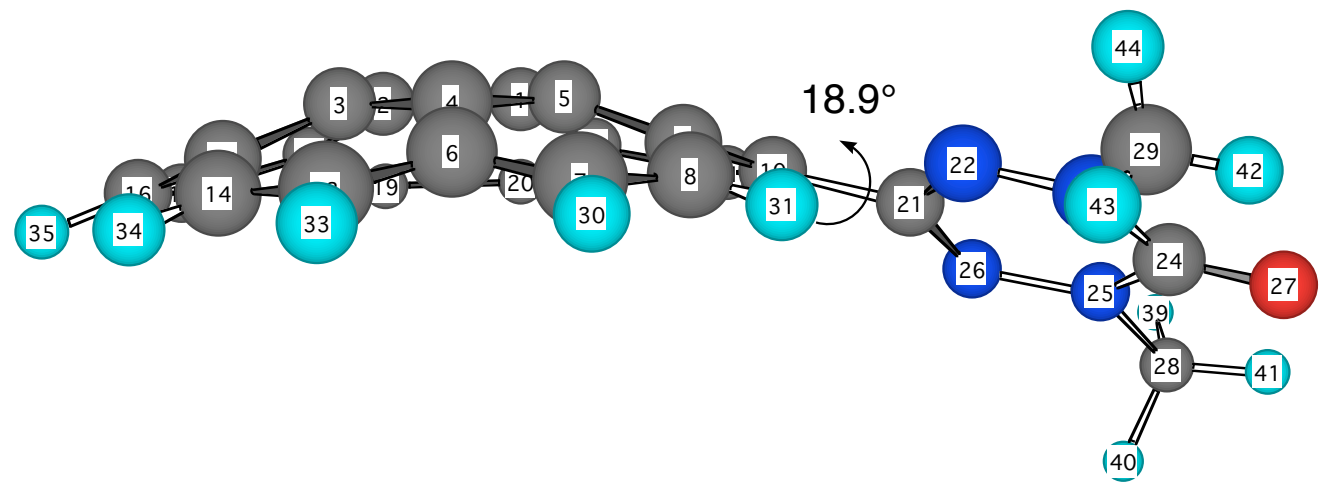

b)

Figure S13. Optimized structures for radical 2 by UBLYP/6-31G** method. (a) top view; (b) side view. 
Gaussian 98 archive file for 2.

$1 ¥ 1 ¥ G I N C-A P L S V ¥ F O p t ¥ U B L Y P ¥ 6-31 G(d, p) ¥ C 24 H 15 N 4 O 1(2) ¥ Y 41103 ¥ 18-J a n-2003 ¥$ 0¥¥\#P UBLYP/6-31G(D,P) NOSYMM OPT SCF=DIRECT DENSITY=CURRENT FORMCHECK ¥¥cor-ver¥¥0,2¥C,-1.5008034711,1.4094379148,-0.8816181197¥C,-2.923799 $9872,1.3655538446,-0.8848609815 ¥ \mathrm{C},-3.3600156062,1.2510983282,0.4660759$ $552 ¥ \mathrm{C}, 2.2049970543,1.2253955228,1.3012201555 ¥ \mathrm{C},-1.049621827,1.3240883$ $486,0.4686209333 ¥ \mathrm{C},-2.1934820752,0.6156338005,2.5575192081 ¥ \mathrm{C},-0.861530$ $1473,0.2853682215,3.0383178067 ¥ \mathrm{C}, 0.2761930158,0.3908833564,2.226500915$ $5 ¥ \mathrm{C}, 0.2076661255,0.8363841291,0.8378261512 ¥ \mathrm{C}, 1.1241615727,0.6236758275$,$0.2996467039 ¥ \mathrm{C}, 0.6515991762,0.6939874593,-1.6297287969 ¥ \mathrm{C},-0.72525866$ $92,0.9929786722,-1.9645024963 ¥ \mathrm{C},-3.5042771276,0.195525744,3.0334817542 ¥ \mathrm{C}$,4.63818802,0.2186540496,2.209992001¥C,-4.5772950574,0.6662678348,0 .8256103233¥C,$5.4552769221,0.3768400344,-0.3000524322 ¥ \mathrm{C},-5.0237276366,0.4861966026,-$ $1.6297871258 ¥ \mathrm{C},-3.6696252303,0.898327195,-1.9709382346 ¥ \mathrm{C}$,$2.8864394823,0.6663675661,-3.1775196364 ¥ C,-1.4858512241,0.7117974446$,$3.1754381312 ¥ \mathrm{C}, 2.5582353867,0.2623818901,-$ $0.086866336 ¥ \mathrm{~N}, 3.0991639706,0.5992690334,1.1041213902 ¥ \mathrm{~N}, 4.4327979904,0.2718500676,1$. $2586397477 ¥ \mathrm{C}, 5.2359350799,-0.3481459309,0.2853894202 ¥ \mathrm{~N}, 4.5322051255,-0.6104908865$, $-0.9052545258 ¥ \mathrm{~N}, 3.1947499714,-0.3468956163,-1.1162005332 ¥ \mathrm{O}, 6.4272598076$,$0.6284581912,0.4539847485 ¥ C, 5.2161803054,-1.277250261,-2.0222673253$ $¥ \mathrm{C}, 5.0122146469,0.6265281065,2.5617798661 ¥ \mathrm{H},-0.7410017439,-0.140443987$ $8,4.0393483843 ¥ \mathrm{H}, 1.2346688022,0.0627324172,2.6275427698 ¥ \mathrm{H}, 1.3593868074$ ,0.4382921493,-2.4206135274¥H,-3.5984648606,-0.236389395,4.0345674854¥ H,$5.5724243115,-0.1964543168,2.6004631547 ¥ \mathrm{H},-6.4554984411,-0.02503968$ 24,$0.1111754476 ¥ \mathrm{H},-5.7041600796,0.1647259605,-2.4242414415 ¥ \mathrm{H},-3.39433$ 06136,0.360154086,-4.0971851864¥H,-0.9545985638,0.4399667605,-4.092502 1051¥H,5.0979162354,-0.6693214207,-2.9311731014¥H,4.7674196119,-2.2679 656761,2.1943902144¥H,6.2740003671,-1.3801413152,-1.7611058005¥H,6.08 $89351905,0.4345601218,2.5189285782 ¥ \mathrm{H}, 4.551096318,0.0178997499,3.355727$ $3782 ¥ \mathrm{H}, 4.8131586448,1.6885554407,2.7652290757 ¥ ¥$ Version=IA64-Linux-G98R evA.11.3¥HF $=-1217.3447848 ¥ S 2=0.758633 ¥ S 2-1=0 . ¥ S 2 A=0.750035 ¥ R M S D=7.244 \mathrm{e}-$ $09 ¥ \mathrm{RMSF}=1.595 \mathrm{e}-06 ¥ \mathrm{Dipole}=-0.1429714,-0.5498485,-0.0948582 ¥ \mathrm{PG}=\mathrm{C} 01[\mathrm{X}(\mathrm{C}$ 24H15N4O1)]¥¥@ 


\section{References}

(S1) (a) Kuhn, R.; Hammer, I. Chem. Ber. 1950, 83, 413-414. (b) Wilmarth, W. K.; Schwartz, N. J. Am. Chem. Soc. 1955, 77, 4543-4551.

(S2) (a) Seider, T. J.; Elliott, E. L.; Grube, G. H.; Siegel, J. S. J. Am. Chem. Soc. 1999, 121,

7804-7813. (b) Rabideau, P. W.; Sygula, A. J. Am. Chem. Soc. 2000, 122, 6323-6324. (c)

Rabideau, P. W.; Xu, G.; Sygula, A.; Marcinow, Z. Tetrahedron Lett. 2000, 41, 9931-9934.

(S3) Seider, T. J.; Elliott, E. L.; Grube, G. H.; Siegel, J. S. J. Am. Chem. Soc. 1999, 121, 7804-7813.

(S4) Neugebauer, F. A.; Fischer, H.; Siegel, R. Chem. Ber. 1988, 121, 815-822. 